\title{
Hollow and Solid Spherical Azithromycin Particles Prepared by Different Spherical Crystallization Technologies for Direct Tableting
}

\author{
Kui Chen ${ }^{1}$, Baohong Hou ${ }^{1,2}{ }^{,}$Hao Wu ${ }^{1}$, Xin Huang ${ }^{1,2}{ }^{\mathbb{D}}$, Fei Li ${ }^{1,2}$, Yan Xiao ${ }^{1}$, Jing Li ${ }^{1}$, \\ Ying Bao ${ }^{1,2}$ and Hongxun Hao ${ }^{1,2, * \mathbb{D}}$ \\ 1 National Engineering Research Center of Industrial Crystallization Technology, \\ School of Chemical Engineering and Technology, Tianjin University, Tianjin 300072, China; \\ chenkui@tju.edu.cn (K.C.); houbaohong@tju.edu.cn (B.H.); hao_wu@tju.edu.cn (H.W.); \\ x_huang@tju.edu.cn (X.H.); lifeijessie@tju.edu.cn (F.L.); yanxiao@tju.edu.cn (Y.X.); lijing@tju.edu.cn (J.L.); \\ yingbao@tju.edu.cn (Y.B.) \\ 2 Collaborative Innovation Center of Chemical Science and Engineering (Tianjin), Tianjin 300072, China \\ * Correspondence: hongxunhao@tju.edu.cn; Tel.: +86-22-27405754
}

Received: 19 April 2019; Accepted: 9 May 2019; Published: 10 May 2019

\begin{abstract}
Many drugs have a propensity for agglomeration, resulting in poor flowability. Spherical crystallization can be used to improve product properties including flowability and particle size. In this work, two methods were developed and utilized to successfully make two kinds of azithromycin spherical particles, namely solid and hollow spheres. The resultant product exhibited regular spherical shape, large particle size, narrow particle size distribution and excellent flowability. The formation mechanism of these different spherical crystals was investigated with the help of a particle vision microscope (PVM). The immersion mechanism and the counter diffusion mechanism were proposed as the formation mechanisms for solid and hollow spheres, respectively. The effects of crystallization parameters on the spherical crystallization processes were investigated systematically. Furthermore, the tablet properties were evaluated to verify that the spherical particles obtained in this work can be directly used for tableting, thus avoiding granulation processes and reducing cost.
\end{abstract}

Keywords: spherical particles; azithromycin; immersion; counter diffusion; powder property

\section{Introduction}

In the pharmaceutical industry, the crystallization process is used not only for separation and purification, but also for improving product properties by controlling crystal habit and polymorph [1-5]. It is widely acknowledged that the flowability, particle size distribution and compressibility of products can greatly influence the downstream operations including filtration, drying, packing and tableting $[6,7]$. Especially, the flake-like and needle-like microcrystals might cause blockage and generate non-uniform tablets in the production process due to their poor flowability and compressibility. In order to solve these problems, the granulation process is commonly used to improve product properties before tableting. However, the granulation process needs specialized devices and lots of excipients, which makes it a time-consuming and costly process. Recently, direct tableting technology without granulation has been brought to attention due to its feasibility of reducing the cost and labor [8-10]. For direct tableting, the products should have outstanding flowability, high compressibility and suitable crystal size to satisfy the strict requirements of the direct tableting process.

Spherical crystallization technology has the potential to address these issues by endowing spherical crystals with excellent powder properties [11,12]. As the crystallization and spherical particles formation are achieved in one single step, the granulation process could be avoided [13]. 
The particles prepared by spherical crystallization usually have a large size which can facilitate the filtration and drying process. Additionally, spherical particles have smaller radius of curvature, thus displaying superior flowability than other morphologies. Furthermore, the dissolution rate of particles can also be efficiently enhanced by spherical crystallization technology, since spherical particles consist of microcrystals without losing their micromeritics property [14]. Therefore, spherical crystallization technology is also an advisable alternative for poorly water-soluble drugs to increase the dissolution rate.

Azithromycin $\left(\mathrm{C}_{38} \mathrm{H}_{72} \mathrm{~N}_{2} \mathrm{O}_{12}\right.$, CAS No. 83905-01-5), a macrolide antibiotic, is widely used to cure urinary and respiratory infections. It is a poorly water-soluble drug and prone to agglomerate during the crystallization process, which leads to low bioavailability and poor flowability [15]. Although many efforts have been made to improve the properties of azithromycin [16,17], a facile method of fabricating spherical crystals has seldom been reported.

In this work, the spherical agglomerate (SA) method was employed to prepare solid azithromycin spherical particles. Generally, three different solvents, namely good solvent, poor solvent, and bridging liquid, were used in this process [18]. The drug was dissolved in the good solvent. The poor solvent will be added into the solution to form tiny precipitates. The bridging liquid which has a good affinity with the precipitates is used to bind the microcrystals together, thus forming the spherical agglomerates. In this work, a modified spherical agglomeration method was proposed. The bridging liquid is directly mixed with the good solvent before adding poor solvent in this method. Ethyl acetate was selected as the bridging liquid, because it is not miscible with water. Emulsion droplets will be formed during crystallization process, and microcrystals can be attracted into these droplets, facilitating the formation of spherical crystals. The working mechanism of this method was also revealed by using process analytical tools (PAT). To further improve the dissolution rate of the model compound, the quasi-emulsion solvent diffusion (QESD) method was developed to prepare hollow spherical particles of azithromycin. In this method, crystallization will firstly occur on the surface of emulsion droplets due to the counter diffusion of solvents on the droplets surface. The formation mechanism of the hollow spherical particles of the QESD method was also investigated in detail.

Additionally, crystallization parameters such as stirring speed, dosage of bridging liquid and temperature were studied to determine the key factors affecting spherical crystallization processes. The powder flowability, packability, purity and residual solvent of spherical products were evaluated and compared with commercial product. Finally, the properties of tablets made of spherical particles were also investigated systematically.

\section{Materials and Methods}

\subsection{Materials}

Azithromycin (purity 99.7\%) was provided by Shijiazhuang Pharmaceutical Co., Ltd. (Shijiazhuang, China). Ethyl acetate, acetone phosphate buffer, acetonitrile, methanol, phosphoric acid and Tween 80 were analytical pure and obtained from Yuan li Chemical Co., Ltd. (Tianjin, China). They were utilized without further purification. Ultrapure water was purified by using a Millipore purification system (model KX2G, Kai xu Co., Ltd, Suzhou, Jiangsu, China). Croscarmellose sodium (purity 99.5\%), sodium lauryl sulfate (purity $99.5 \%$ ), polyethylene glycol (purity 99.8\%), magnesium stearate (purity 99.9\%) and microcrystalline cellulose (purity 99.8\%) were purchased from Jiang Tian Chemical Co., Ltd. (Tianjin, China).

\subsection{Preparation of Spherical Crystals}

\subsubsection{SA Method for the Preparation of Solid Spherical Crystals}

Azithromycin (12 g) was fully dissolved in $30 \mathrm{~mL}$ mixture of acetone (good solvent) and ethyl acetate (bridging liquid) with volume ratio of 2:1 in a jacketed crystallizer which was connected with a 
thermostat to keep the system temperature constant at $30^{\circ} \mathrm{C}$. The agitator, a two-bladed propeller, was set at $700 \mathrm{rpm}$. Then, about $90 \mathrm{~mL}$ deionized water (poor solvent) was slowly added into the mother liquid at the rate of $1 \mathrm{~mL} / \mathrm{min}$ by a peristaltic pump. With the addition of water, spherical particles gradually formed and grew up. After the water was completely added, the system was continuously stirred for $30 \mathrm{~min}$. Finally, the spherical crystals were filtered, washed with $30 \mathrm{~mL}$ water and dried at $50{ }^{\circ} \mathrm{C}$ for $5 \mathrm{~h}$ in a vacuum oven.

\subsubsection{QESD Method for the Preparation of Hollow Spherical Crystals}

Excess azithromycin $(8 \mathrm{~g})$ was added into $10 \mathrm{~mL}$ ethyl acetate at $50{ }^{\circ} \mathrm{C}$ to prepare saturated solution. Then, $0.2 \mathrm{~g}$ Tween 80 , selected as the emulsifier, was added into $100 \mathrm{~g}$ deionized water. The system temperature was controlled at $20^{\circ} \mathrm{C}$ and the stirring speed was set at $200 \mathrm{rpm}$. Then, $5 \mathrm{~g}$ saturated azithromycin solution was added into the aqueous solution dropwise. After agitating for $30 \mathrm{~min}$, the spherical crystals were filtered, washed with $50 \mathrm{~mL}$ water and dried at $50{ }^{\circ} \mathrm{C}$ for $5 \mathrm{~h}$ in a vacuum oven (DZ2B, Tianjin Taisi Co., Ltd, Tianjin, China).

\subsection{Characterization of Products}

An optical microscope (Eclipse E200, Nikon, Tokyo, Japan) and scanning electron microscope (TM3000, Hitachi, Tokyo, Japan) were used to observe the morphologies of the crystals. Besides, the process of spherical crystallization was monitored by particle visual microscope (PVM) (V819, Mettler, Zurich, Switzerland).

Powder X-ray diffractometer (PXRD) (D/max2500, Rigaku, Tokyo, Japan), $\mathrm{Cu}$ K $\alpha$ radiation $(\lambda=1.5418 \AA, 40 \mathrm{kV} \times 100 \mathrm{~mA})$, was utilized for polymorphic characterization. The date was collected in diffraction angle $(2 \theta)$ from $2^{\circ}$ to $40^{\circ}$ at the scanning rate of $0.067^{\circ} \cdot \mathrm{s}^{-1}$.

\subsection{Determination of Spherical Crystal Properties}

\subsubsection{Flowability}

The angle of repose was measured to evaluate the flowability of the spherical crystals, using the fixed funnel method $[19,20]$. The tap density (TD) was obtained by tapping the samples in a cylinder using a tapping machine. Bulk density $(B D)$ was calculated by the volume and weight of the powder before tapping. Carr's index $(C I)$ and Hausner's ratio (HR) was calculated according to Equations (1) and (2), respectively:

$$
\begin{gathered}
C I=\frac{(T D-B D)}{T D} \times 100, \\
H R=\frac{T D}{B D} .
\end{gathered}
$$

An angle of repose below $25^{\circ}$ indicates good flow property whereas that above $40^{\circ}$ reflects poor flowability. CI below 15 or $H R$ below 1.35 indicates good flowability, while $C I$ above 35 or $H R$ above 1.35 represents poor flowability [21].

\subsubsection{Particle Size Distribution (PSD)}

The PSD was measured by Mastersizer 3000 (British Malvern instrument Co. Ltd, Malvern, Worcestershire, United Kingdom), using water as the dispersant. The value of span indicates the width of PSD, which was calculated according to Equation (3) [22].

$$
\text { Span }=\frac{D_{90}-D_{10}}{D_{50}}
$$

where $D_{10}, D_{50}$ and $D_{90}$ represent the particle sizes at the point of $10 \%, 50 \%$ and $90 \%$ cumulative volume fraction. 


\subsubsection{Packability}

Improving the packability of the drug is beneficial to conduct tableting. The packability was evaluated by Kawakita and Kuno' equations [23]:

$$
\begin{aligned}
& \frac{n}{c}=\frac{n}{a}+\frac{1}{a b}, \\
& c=\frac{V_{0}-V_{n}}{V_{0}},
\end{aligned}
$$

where $n$ is the tap times, $1 / a$ defines the degree of volume reduction after being taped $n$ times, and parameter $b$ can indicate the packing velocity [24]. The smaller value of parameter $a$ is, the better the packability is. Parameter $c$ denotes the volume reduction which can be calculated from Equation (5). $V_{0}$ and $V_{n}$ correspond to the initial volume and final volume after being taped $n$ times, respectively.

\subsubsection{Mensuration of Purity and Residual Solvents}

High-performance liquid chromatography (Agilent 1200 HPLC, Agilent Co., Ltd, Palo Alto, CA, USA) equipped with a C18 column $(250 \times 4.6 \mathrm{~mm}, 5 \mu \mathrm{m})$ was used to measure the purity of azithromycin. The mixed solvent of $60 \%(v / v)$ phosphate buffer, $20 \%(v / v)$ acetonitrile and $20 \%(v / v)$ methanol was utilized as mobile phase which was adjusted to $\mathrm{pH} 8.0$ with phosphoric acid. The flow rate was $1.0 \mathrm{~mL} / \mathrm{min}$, and the volume of each injection was $20 \mu \mathrm{L}$. UV detection was set at $\lambda_{\max }$ of $215 \mathrm{~nm}$.

The residual organic solvents of azithromycin were measured by gas chromatography (HP 4890, Agilent Co., Ltd, Palo Alto, CA, USA). A capillary column $(30 \mathrm{~m} \times 0.53 \mathrm{~mm} \times 0.25 \mu \mathrm{m})$ was employed. The residual water was measured by a Karl Fischer titrator (DL 18, Mettler, Switzerland).

\subsection{Determination of Tablet Properties}

\subsubsection{Preparation of Tablets}

The azithromycin tablets were prepared according to the formulation in the reported literature [25]. Azithromycin tablet (total mass $500 \mathrm{mg}$ ) contained 40\% (w/w) azithromycin, $5 \%(w / w)$ croscarmellose sodium, $0.5 \%(w / w)$ Sodium lauryl sulfate, $20 \%(w / w)$ Polyethylene glycol, $2 \%(w / w)$ magnesium stearate and $32.5 \%(w / w)$ Microcrystalline Cellulose. After adequately mixing, a rotary tablet press (ZP10A, Shanghai Tianqi Pharmaceutical Machinery Co., Ltd, Shanghai, China) was employed to prepare tablets.

\subsubsection{Compactibility}

Compaction curves was measured by plotting the tensile strength versus pressure. Tablets' tensile strength can be calculated by the following Equation (6):

$$
\text { Tensilestrength }=\frac{2 F}{\pi d h},
$$

where $F$ represents the force breaking the tablet, $h$ and $d$ are the thickness and diameter of tablet, respectively.

\subsubsection{Friability}

The initial weight of 20 tablets were measured at first. Then, these tablets were placed in the tumbling chamber of a friability apparatus (TAR 10, Erweka, Düsseldorf, Nordrhein-Westfalen, Germany), and rotated at $25 \mathrm{rpm}$ for $4 \mathrm{~min}$. Finally, the 20 tablets were weighed again. The friability was calculated by the Equation (7). 


$$
\text { Friability }=\frac{\text { Initial weight }- \text { Final weight }}{\text { Initial weight }} \times 100 \%
$$

\subsubsection{Disintegration Time}

Six tablets were randomly selected to measure the disintegration time by putting one tablet in each tube, using deionized water as the disintegration medium in a disintegration test apparatus (BJ-3, Guo ming Pharmaceutical Co., Ltd, Tianjin, China) at $37 \pm 0.5^{\circ} \mathrm{C}$. When the tablets completely disintegrated and passed through the mesh, the disintegration time was recorded and average disintegration time of the six tablets was calculated.

\subsubsection{Content Uniformity}

To determine the content uniformity, 10 tablets were placed separately in a $250 \mathrm{~mL}$ volumetric flask, then deionized water was added to $250 \mathrm{~mL}$. After the tablet was dissolved entirely, the sample was filtered by $0.2 \mu \mathrm{m}$ microporous membrane and analyzed by HPLC.

\subsubsection{In Vitro Dissolution Experiment}

The dissolution rate was measured in US Pharmacopeia Apparatus II (DT6R, Erweka, Düsseldorf, Nordrhein-Westfalen, Germany) [26]. A total of $900 \mathrm{~mL}$ phosphate buffer (pH 6.0) and $0.1 \mathrm{~mol} / \mathrm{L} \mathrm{HCl}$ solution were used as the dissolution medium, respectively. One tablet was added into the medium at $37 \pm 0.5^{\circ} \mathrm{C}$ and agitation of $100 \mathrm{rpm}$. Then, $5 \mathrm{~mL}$ samples were taken out at time intervals of 5,10 , $15,20,30,40,60,80,100$ and $120 \mathrm{~min}$. Samples were filtered by $0.2 \mu \mathrm{m}$ microporous membrane and analyzed by HPLC.

\section{Results and Discussion}

\subsection{Characterization of Spherical Products}

As shown in Figure 1, the commercial azithromycin has a non-uniform particle size and agglomerates seriously, which will cause tableting difficulty in the downstream processes. Figure 2a,c shows the SEM images of solid spheres and hollow spheres prepared by SA and QESD methods in this work. Clearly, the spherical crystals possess large particle size, regular spherical shape and smooth surface which would benefit the tableting of the final products. Furthermore, their different interior structures are clearly observed in Figure $2 \mathrm{~b}, \mathrm{~d}$. Microcrystals agglomerated into solid spheres, and hollow spheres had a large cavity and porous structure. The structure units of spherical particles are shown in Figure S1 in the Supporting Information. It was found that the size of single particle is about $30 \mu \mathrm{m}$, and some submicro-sized particles were also embedded in the spherical particles. Besides, as shown in Figure 3a, the spherical products prepared by both methods present narrow and unimodal size distribution, demonstrating that the size of spherical particles was uniform. On the contrary, the commercial product exhibits a broad and bimodal size distribution.

For the azithromycin, the desired form is the azithromycin dihydrate due to its excellent stability. However, the monohydrate crystal usually precipitates first and then transforms into dihydrate [27]. Therefore, it is necessary to verify whether polymorphism or solvate transformation happens during the spherical crystallization processes. According to the PXRD diffraction patterns in Figure $3 b$, all the products are azithromycin dihydrate and no polymorphic transformation happened during the crystallization processes. Hence, the desired crystal form can be directly obtained in the spherical crystallization process, ensuring the uniformity in crystal forms. The reason might be that the azithromycin dihydrate is both thermodynamically and kinetically favored in the presence of ethyl acetate. 


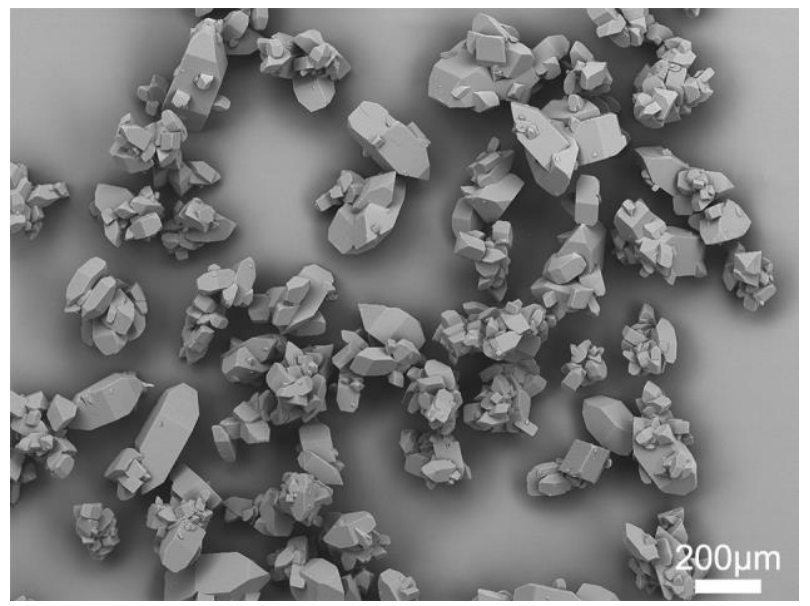

Figure 1. SEM image of commercial azithromycin.
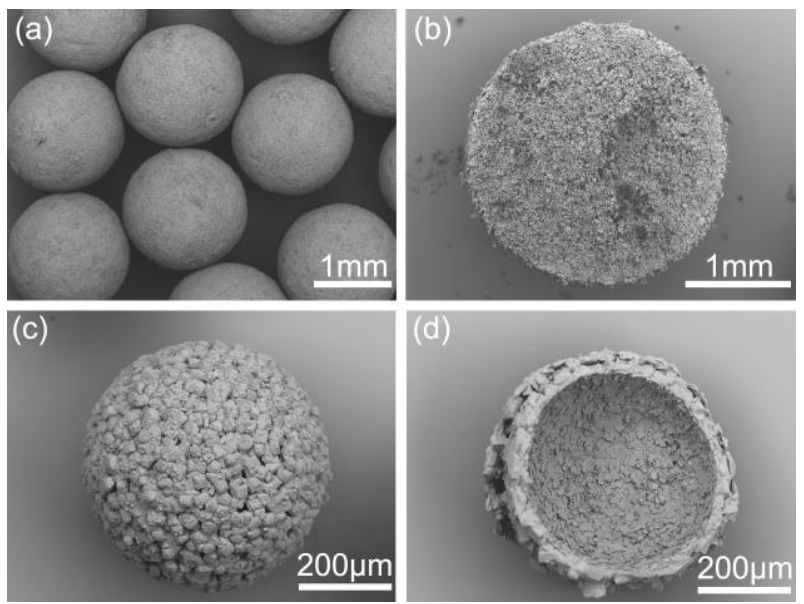

Figure 2. SEM images of different products: (a) crystals prepared by the spherical agglomerate (SA) method, (b) profile chart of solid sphere particle; (c) crystals prepared by the quasi-emulsion solvent diffusion (QESD), (d) profile chart of hollow sphere particle.
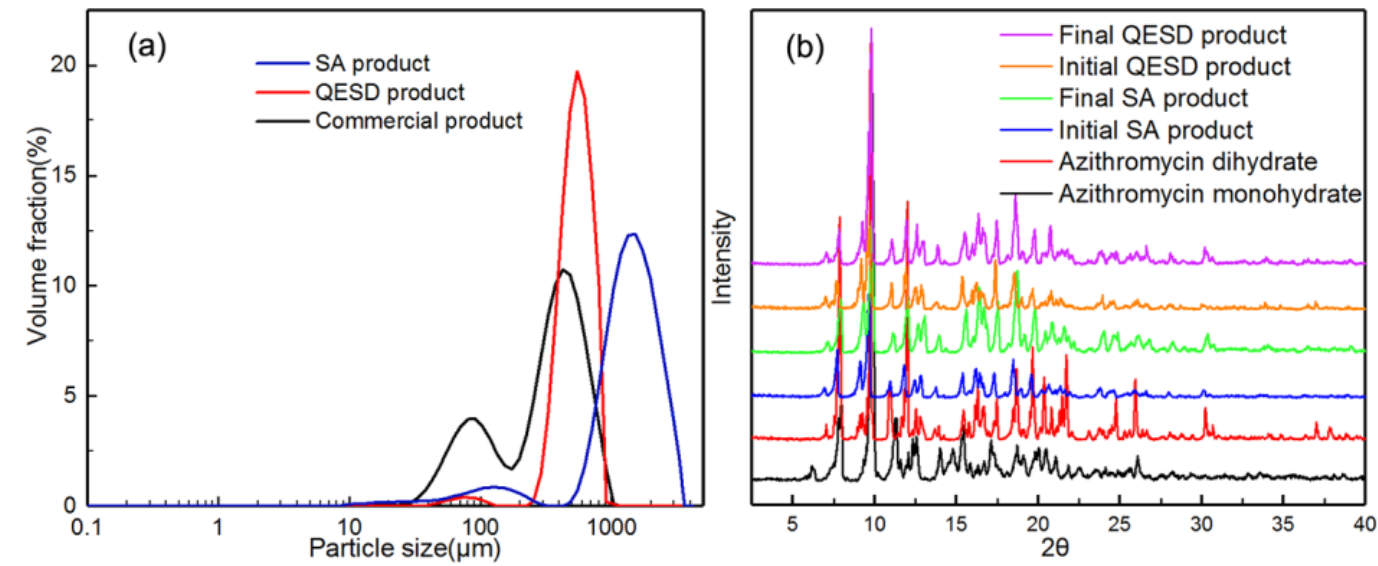

Figure 3. (a) Particle size distribution of the commercial and spherical products; (b) PXRD diffraction patterns of the crystallization products. 


\subsection{Formation Mechanisms of Spherical Crystals}

\subsubsection{SA Process}

As shown in Figure 4, the formation mechanism of the solid spheres was proposed. To confirm this hypothesis, online PVM was used to in situ monitor the process and an optical microscope was used as assistant tool. The results are shown in Figure 5.

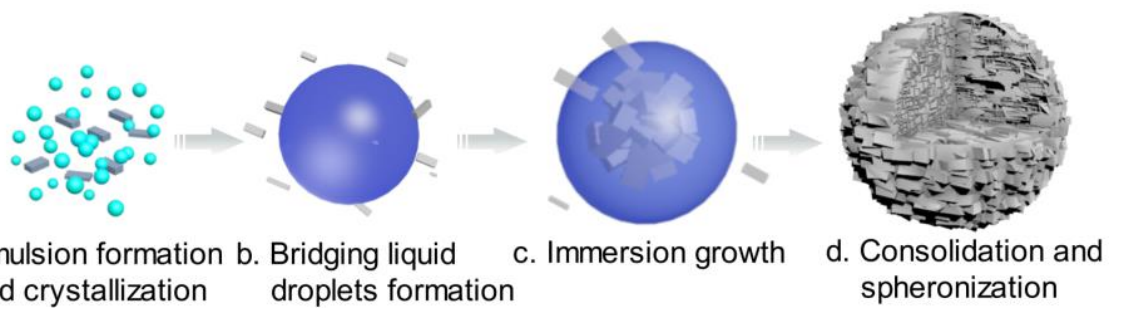

Figure 4. Mechanism schematic of spherical agglomeration (cyan: water; blue: bridging liquid; gray: crystal).
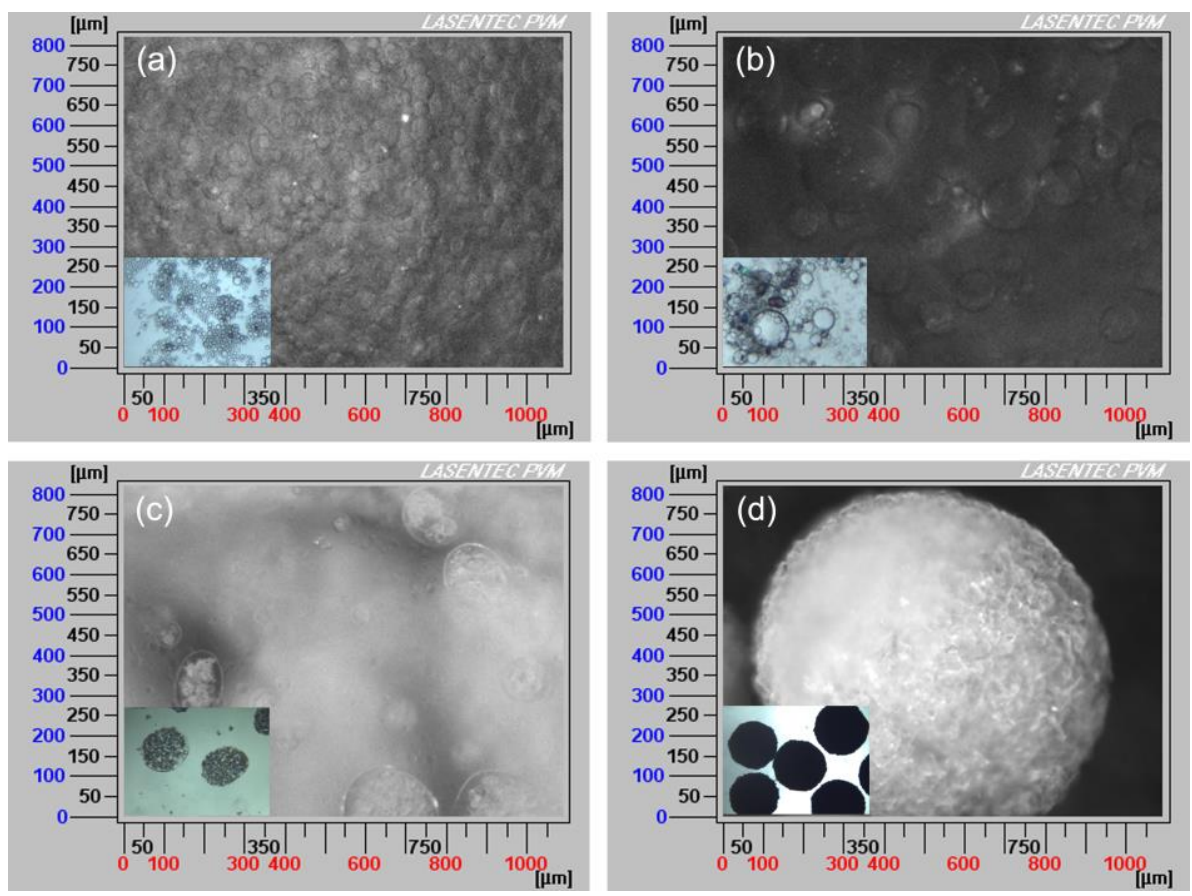

Figure 5. Particle visual microscope (PVM) images of the spherical agglomeration process with the addition of different volumes of water: (a) $15 \mathrm{~mL}$, (b) $25 \mathrm{~mL}$, (c) $45 \mathrm{~mL}$, (d) $90 \mathrm{~mL}$ (insets: optical microscope images).

When an appropriate volume water was added, some crystals appeared and water/oil (W/O) emulsion droplets were also formed since water is immiscible with the bridging liquid, as shown in Figures $4 \mathrm{a}$ and $5 \mathrm{a}$. With further addition of water, the dispersed phase was changed, hence bridging liquid droplets were formed (Figures $4 b$ and $5 b$ ) and the size of droplets became large. Due to the affinity of bridging liquid, small single crystals would be immersed into the bridging liquid droplets and agglomeration will occur [28-30], facilitating the growth of spherical particles, as seen in Figures $4 \mathrm{c}$ and $5 \mathrm{c}$. Since the bridging liquid was excess, the initial agglomerates looked like paste and were surrounded by bridging liquid [31]. Continuously adding water, more crystals would be attracted to the bridging liquid and hence agglomerates will become larger. Then bridging liquid gradually diffused into the continuous phase with the addition of water, leading to the disappearance of the layer of bridging liquid and consolidation of spherical particles. Meanwhile, due to the shear force and collision, robust, uniform, large and regular spherical solid particles were finally obtained (Figures $4 \mathrm{~d}$ and $5 \mathrm{~d}$ ). 


\subsubsection{QESD Process}

The formation schematic and PVM images of hollow sphere crystals are shown in Figures 6 and 7. When the saturated ethyl acetate solution was dropwise added into the aqueous phase, the oil/water $(\mathrm{O} / \mathrm{W})$ emulsion droplets were formed, as displayed in Figures 6a and 7a. Meanwhile, as the ethyl acetate gradually diffused out of the emulsion droplets and the water diffused into the emulsion droplets (Figure 6b), azithromycin rapidly nucleated and precipitated on the surface of the droplets surface, as shown in Figures $6 \mathrm{c}$ and $7 \mathrm{~b}$. When ethyl acetate diffused into the aqueous phase entirely, more crystals precipitated on the surface of droplets and a hollow sphere was formed (Figure 6d). In Figure $7 d$, the hollow structure is clearly observed. Therefore, the counter diffusion of ethyl acetate and water on the surface of droplets is the main formation mechanism of hollow spherical crystals. Although many studies have focused on spherical particles, most of them are porous or loose [32]. However, in this work, tight hollow spherical crystals were successfully obtained via a facile method. The formation of the special structure can be attributed to the fact that azithromycin is extremely insoluble in water. Azithromycin could precipitate quickly on the surface of the droplets when saturated ethyl acetate solution was added into the aqueous phase. Besides, suitable concentration, temperature and solvent are also the critical factors for the formation of hollow structure.

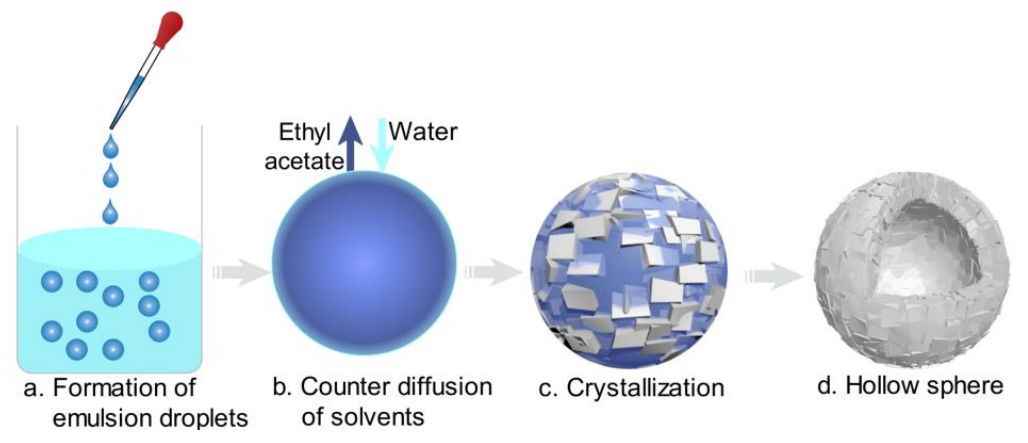

Figure 6. Mechanism schematic of hollow spherical crystals (cyan: water; blue: bridging liquid; gray: crystal).
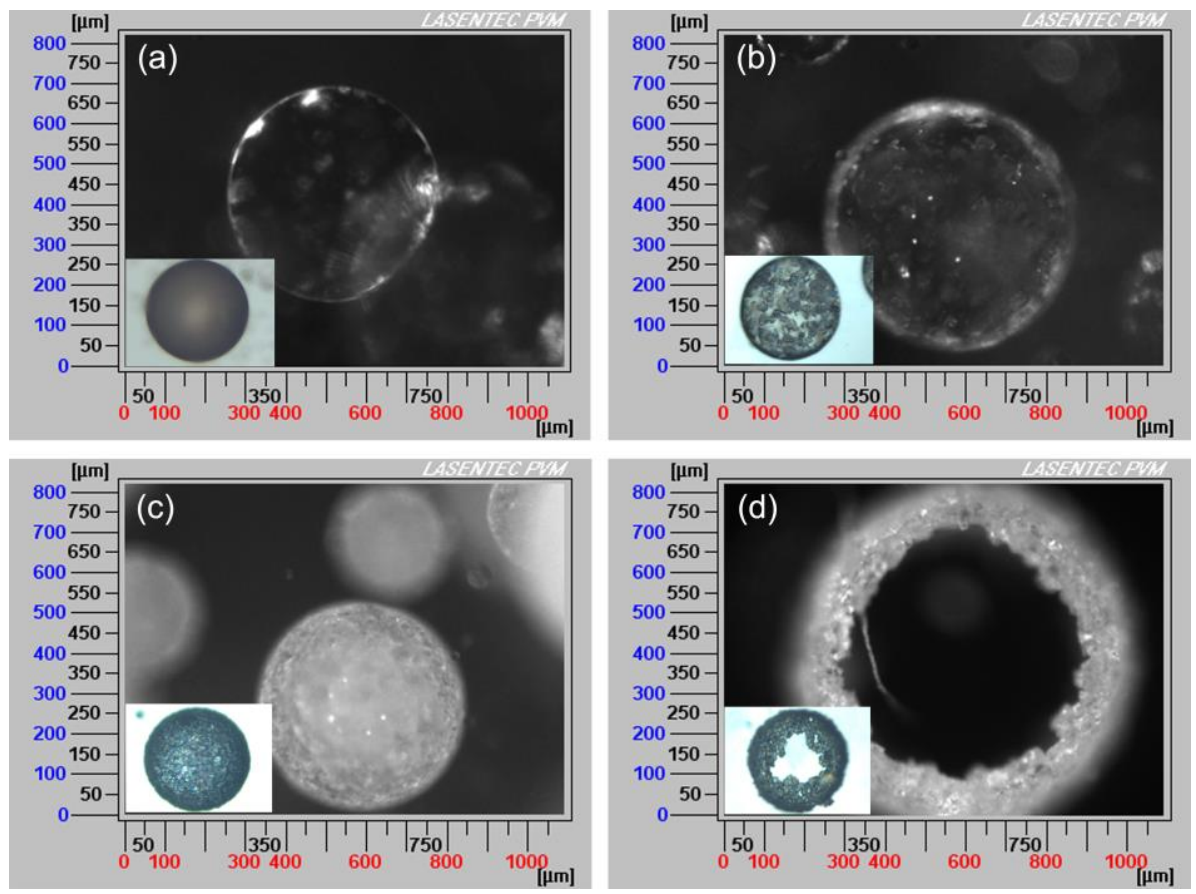

Figure 7. PVM images of the QESD process with the addition of saturated ethyl acetate solution at (a) $0 \mathrm{~min}$, (b) $1 \mathrm{~min}$, (c) $5 \mathrm{~min}$ and (d) $30 \mathrm{~min}$, respectively (insets: optical microscope images). 


\subsection{Effect of Process Parameters on Spherical Crystallization Processes}

As mentioned above, crystallization conditions have crucial effects on spherical crystallization processes. To optimize the spherical crystallization process, the effects of different crystallization conditions were investigated.

\subsubsection{Effect of Crystallization Parameters on SA Process}

For the spherical agglomeration, the stirring speed $(\mathrm{N})$, dosage of bridging liquid $\left(\mathrm{V}_{\mathrm{b}}\right)$ and concentration of substance $\left(\mathrm{C}_{\mathrm{s}}\right)$ are the main factors affecting the formation and size of spherical crystals. The experiment conditions are listed in Table S1 in the Supporting Information.

(1) Effect of stirring speed

As presented in Figure 8, spherical crystals with different mean size from 0.51 to $3.73 \mathrm{~mm}$ were prepared at different stirring speeds in experiments SA-1-SA-4. The mean size decreased dramatically with the increase of the stirring speed due to the enhancement of shear force. Hence, the size of spherical particles is easily controlled just by adjusting stirring speed. While, irregular spherical particles were formed at the stirring speed of $1000 \mathrm{rpm}$ in Figure $8 \mathrm{~d}$ due to the destruction of high shear force. Therefore, to obtain large and regular spherical crystals, the stirring speed should be controlled below $1000 \mathrm{rpm}$.
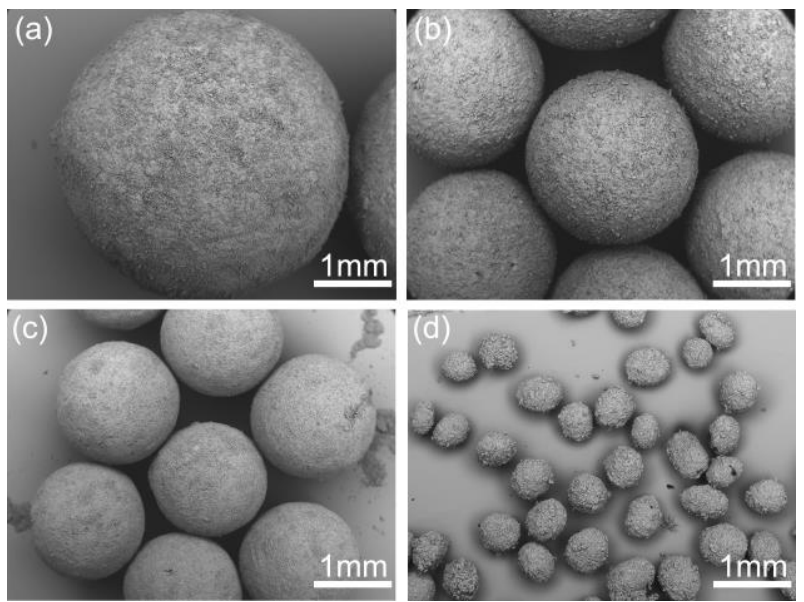

Figure 8. SEM images of the spherical crystals prepared at different stirring speeds: (a) $300 \mathrm{rpm}, \mathrm{SA}-1$; (b) $500 \mathrm{rpm}, \mathrm{SA}-2$; (c) $700 \mathrm{rpm}, \mathrm{SA}-3$; (d) $1000 \mathrm{rpm}, \mathrm{SA}-4$.

(2) Effect of the dosage of bridging liquid

The effect of dosage of bridging liquid on the quality of products are shown in Figure 9. The dosage of bridging liquid apparently affects the formation of spherical crystals. As shown in Figure 9a, due to the lack of the bridging liquid, the spherical particles became irregular and rough. However, the agglomerates became large and smooth with the increase of the dosage of bridging liquid in an appropriate range from 8 to $12 \mathrm{~mL}$. When excessive bridging liquid was added, the system was still in phase separation. Hence, the crystals could not aggregate into spheres and remained paste-like, as shown in Figure 9d. The spherical crystals should be prepared within a critical range. Above the range, a paste-like product will form, while crystals cannot aggregate into spheres below the range. 

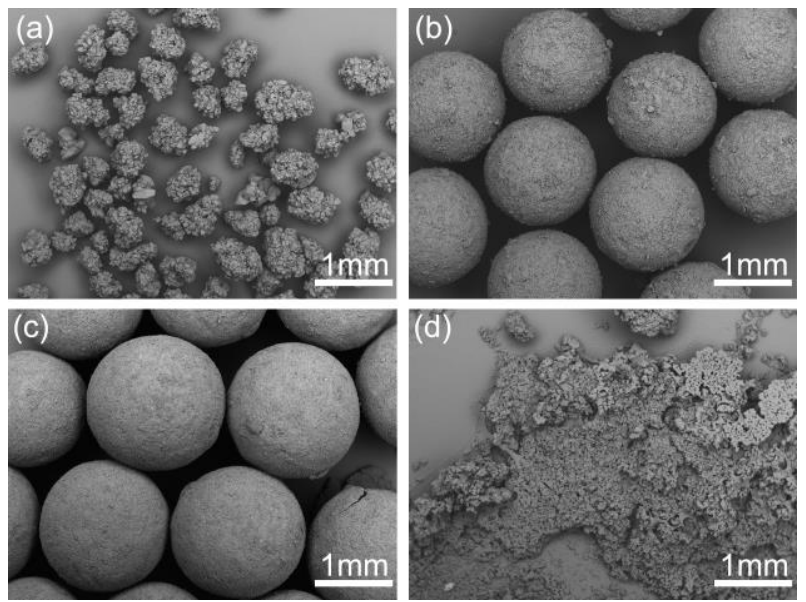

Figure 9. SEM images of the spherical crystals prepared at different dosages of bridging liquid (a) $6 \mathrm{~mL}$, SA-5; (b) 8 mL, SA-6; (c) 12 mL, SA-7; (d) 15 mL, SA-8.

(3) Effect of the concentration of azithromycin

Figure 10 illustrates the influence of the concentration of azithromycin on the spherical crystallization process. It is clear that the size of the spherical crystals increased with the increasing of concentration. At high concentration, azithromycin could rapidly nucleate, and many small crystals which are easier to aggregate together than large crystals will be generated. This is because the adhesive force between the bridging liquid and small crystals is stronger than that between the bridging liquid and larger crystals [33]. However, when the concentration was too high, the surface of spherical crystals (Figure 10d) became rough owing to the lack of bridging liquid.
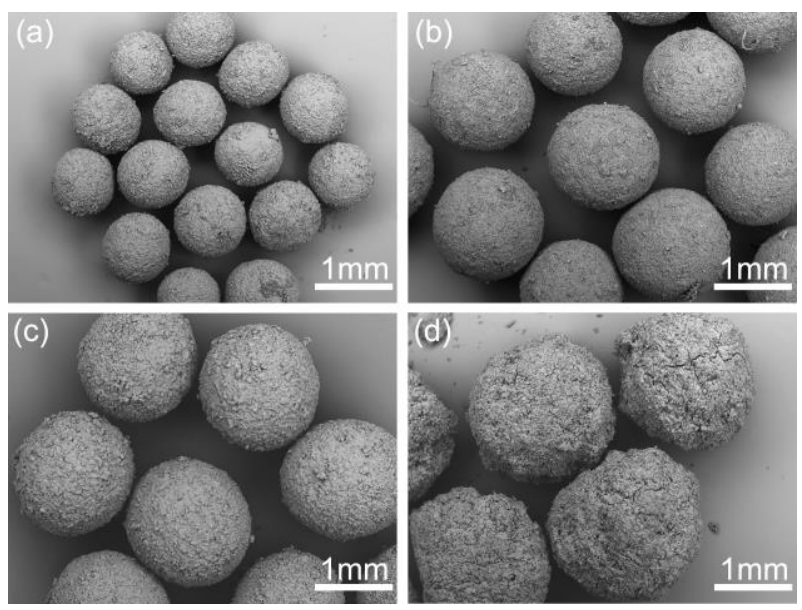

Figure 10. SEM images of the spherical crystals prepared at different concentrations: (a) $0.3 \mathrm{~g} / \mathrm{mL}, \mathrm{SA}-9$; (b) $0.5 \mathrm{~g} / \mathrm{mL}, \mathrm{SA}-10$; (c) $0.6 \mathrm{~g} / \mathrm{mL}, \mathrm{SA}-11$; (d) $0.7 \mathrm{~g} / \mathrm{mL}, \mathrm{SA}-12$.

\subsubsection{Effect of Crystallization Parameters on QESD Process}

In the QESD method, temperature of aqueous phase $(T)$, mass fraction of emulsifier $\left(\omega_{\mathrm{e}}\right)$, concentration of substance $\left(C_{s}\right)$ and stirring speed $(N)$ are the main factors which will affect the formation and size of spherical crystals. The crystallization parameters are listed in Table S2 in the Supporting Information.

(1) Effect of the temperature of aqueous phase

Figure 11 shows the influence of aqueous temperature on the structure of spherical crystals. It can be revealed that the cracked spherical particles appeared at 5 and $40{ }^{\circ} \mathrm{C}$, as described in Figure $11 \mathrm{a}$, d. As the diffusion rate of solvent was slow at $5{ }^{\circ} \mathrm{C}$, the spherical particles could not solidify completely 
in time and were broken by shear force. On the other hand, when the temperature was increased to $40{ }^{\circ} \mathrm{C}$, the solubility of azithromycin will increase, resulting in the decrease of supersaturation level. As a result, the azithromycin could not rapidly precipitate to form a solidified layer and wrap the spherical droplet fast enough. Then, the soft emulsion droplets were easily divided into smaller spherical particles or broken under the shear force and collision. Therefore, the temperature should be controlled within an appropriate range from 10 to $40^{\circ} \mathrm{C}$.
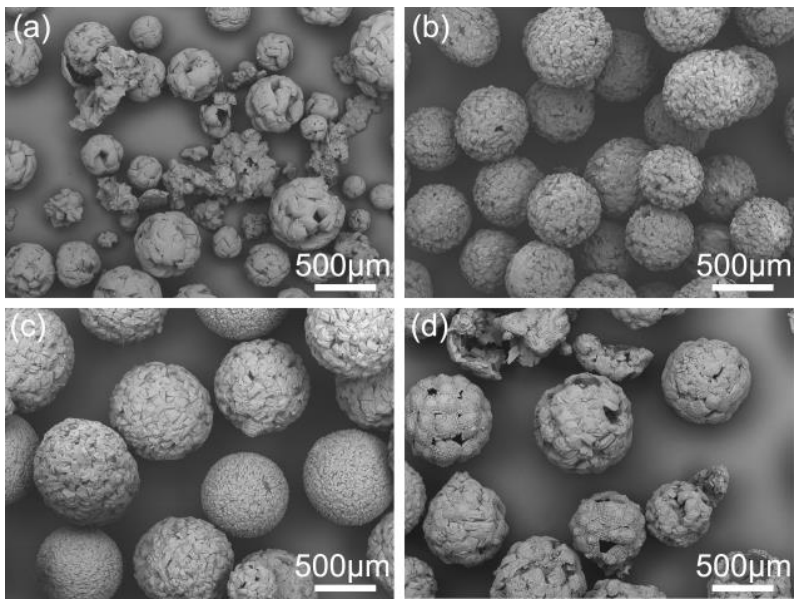

Figure 11. SEM images of spherical crystals prepared at different aqueous temperatures: (a) $5{ }^{\circ} \mathrm{C}$, QESD-1; (b) $20{ }^{\circ} \mathrm{C}$, QESD-2; (c) $30{ }^{\circ} \mathrm{C}$, QESD-3; (d) $40{ }^{\circ} \mathrm{C}$, QESD-4.

(2) Effect of the stirring speed

Stirring speed is also a vital parameter for QESD method. As described in Figure 12, the mean sizes clearly decreased with the increasing of stirring speed. At $600 \mathrm{rpm}$, the high shear force could break the particles into fragments. However, it was not because the hollow spheres were not hard enough. To verify it, the particles were allowed to completely solidify at a low stirring speed of $200 \mathrm{rpm}$, and then the stirring speed was increased to $600 \mathrm{rpm}$. The solidified particles could still remain intact after stirring for $30 \mathrm{~min}$. Therefore, the destruction mainly occurred during the solidification process and the completely solidified hollow spheres were hard enough to resist the friction and collision during production process. Reducing the stirring speed caused the coalescence of particles (Figure 12a), and the particle size was non-uniform due to the lack of shear force. Therefore, the stirring speed should be controlled at a suitable range among 200-600 rpm.
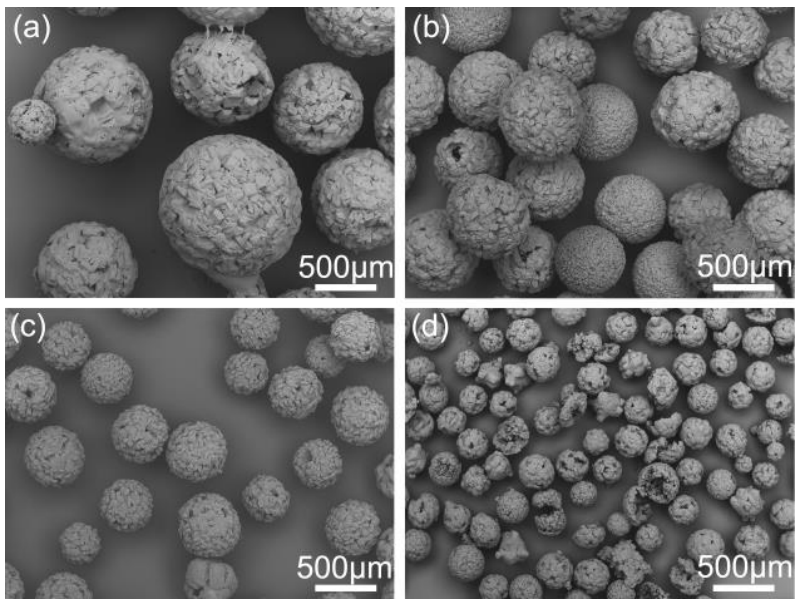

Figure 12. SEM images of the spherical crystals prepared at different stirring speeds: (a) $150 \mathrm{rpm}$, QESD-5; (b) 300 rpm, QESD-6; (c) 400 rpm, QESD-7; (d) 600 rpm, QESD-8. 
(3) Effect of the dosage of the emulsifier

The effects of different dosages of the emulsifier on the spherical crystal size and shape were evaluated. Without the emulsifier (Figure 13a), spherical crystals could not form due to the instability of the emulsion droplets. In the presence of the emulsifier, the emulsion droplets could stay stable and hence regular spherical crystals could form. Furthermore, the size of particles decreased slightly with the increasing dosage of the emulsifier since the interfacial tension declined with the increase of emulsifier concentration.
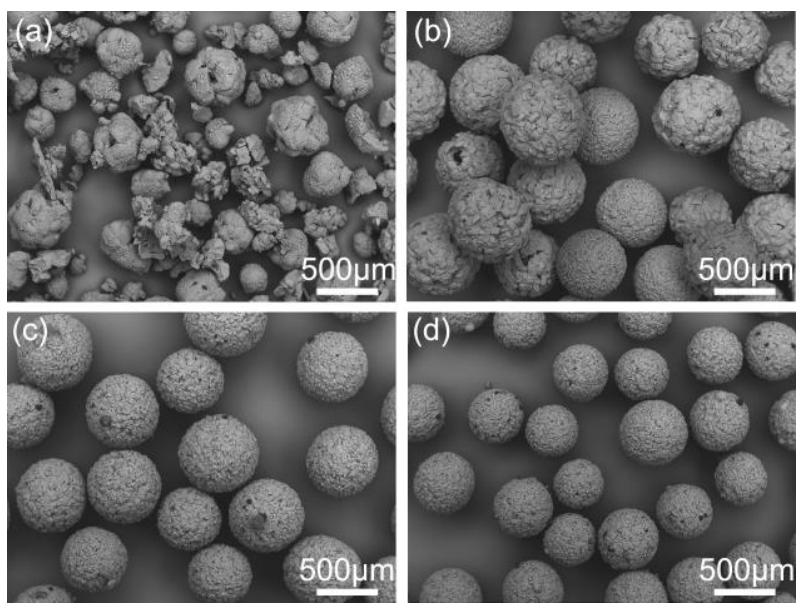

Figure 13. SEM images of the spherical crystals prepared at different concentrations of the emulsifier:

(a) 0\%, QESD-9; (b) 0.2\%, QESD-10; (c) 0.5\%, QESD-11; (d) 2\%, QESD-12.

(4) Effect of the concentration of substance

As shown in Figure 14, the concentration of the substance had an important influence on the size and shape of the spherical particles. With the reduction of the concentration, the size of the spherical particles decreased, and the number of broken particles increased dramatically. It can be explained by the fact that azithromycin could not rapidly precipitate and the number of precipitated crystals will decrease at low concentration. Hence, spherical particles could not solidify in time, which caused the soft spherical particles to be divided into small particles or broken due to the shear force and collision. Therefore, the concentration of substance should be controlled above $0.4 \mathrm{~g} / \mathrm{mL}$.
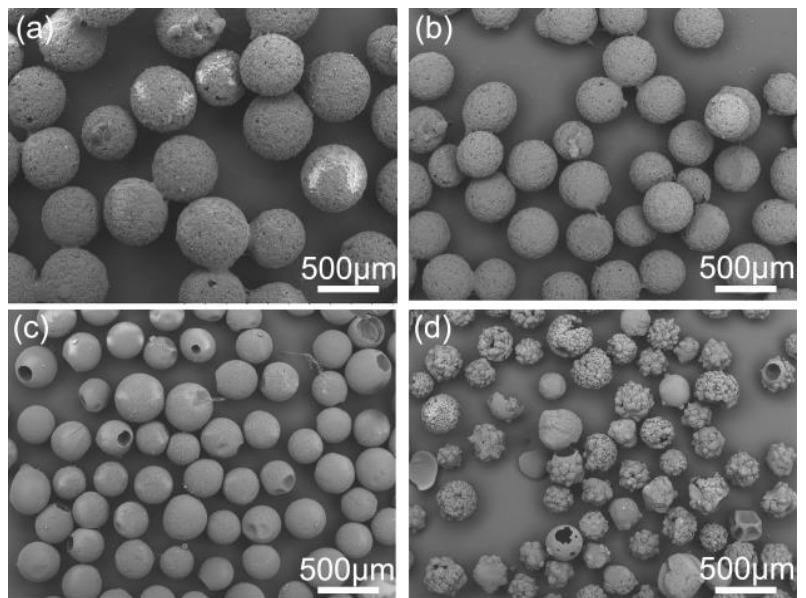

Figure 14. SEM images of the spherical crystals prepared at different concentrations of substance: (a) $0.7 \mathrm{~g} / \mathrm{mL}$, QESD-13; (b) $0.5 \mathrm{~g} / \mathrm{mL}$, QESD-14; (c) $0.4 \mathrm{~g} / \mathrm{mL}$, QESD-2; (d) $0.2 \mathrm{~g} / \mathrm{mL}$, QESD-16.

According to the above analysis, the effects of operating parameters on spherical crystallization processes can be summarized. Dosages of the bridging liquid and emulsifier are the crucial factors 
affecting the formation of solid and hollow spheres, respectively. Stirring speed dramatically affects the size of the spherical particles for the both processes. It is also effective to control the size of spherical crystals by altering the stirring speed. The concentration of the substance has an effect on the size of the spherical particles. Additionally, the temperature significantly affects the intactness of the hollow sphere. In summary, appropriate crystallization parameters are essential to obtain the desired spherical particles.

\subsection{Evaluation of Spherical Crystal Properties}

The properties of spherical products including powder flowability, packability and purity and residual solvent, were evaluated. The products in experiment SA-4 and QESD-7 were selected for the evaluation of different properties.

\subsubsection{Flowability}

In order to make uniform tablets, the raw materials must flow smoothly into the die cavity of the tablet machine. Thus, good flowability is crucial for tableting. To eliminate the influence of particle size on flowability, all the products were sieved through 35- and 40-mesh sieves before use. As listed in Table 1 , the angle of repose of commercial product was $42.7^{\circ}$, which showed extremely poor flowability. However, the spherical products displayed superior flowability than the commercial one and their repose angles were below the standard value of good flowability $\left(25^{\circ}\right)$. Their $C I$ and $H R$ values were significantly lower than the commercial product. Therefore, the flowability of the model compound was significantly improved by spherical crystallization methods. The obtained spherical products are suitable for direct tableting and capsule filling without additional granulation process.

Table 1. Comparison of the commercial and spherical products.

\begin{tabular}{ccccc}
\hline Product Type & $\begin{array}{c}\text { Angle of Reponse } \\
\text { (deg) }\end{array}$ & CI & HR & Mean Size (mm) \\
\hline Commercial & 42.7 & 19.5 & 1.19 & 0.473 \\
product & 19.5 & 6.67 & 1.07 & 0.481 \\
SA product & 24.2 & 7.14 & 1.08 & 0.478 \\
QESD product & & & & \\
\hline
\end{tabular}

\subsubsection{Packability}

The plot of $n / c$ versus $n$ is shown in Figure S2 in the Supporting Information. According to the results of linear fitting, the packability parameters $a, b$ and correlation coefficient $\left(R^{2}\right)$ were calculated and the results are shown in Table 2. Compared to the commercial product, the values of parameter $a$ of spherical products are lower, indicating a higher packability. The values of parameter $b$ represent the packing velocity of tapping. The higher values of $b$ demonstrated that the packing velocity of spherical products is faster than that of the commercial products. Owing to their excellent packability and flowability, the spherical products could be packed closely without tapping many times. Therefore, the production efficiency can be improved in the process of direct tableting and capsule filling.

Table 2. Parameters of packability of the commercial and spherical products.

\begin{tabular}{cccc}
\hline Product Type & $\boldsymbol{a}$ & $\boldsymbol{b}$ & $\boldsymbol{R}^{\mathbf{2}}$ \\
\hline Commercial product & 0.3731 & 0.0427 & 0.9978 \\
SA product & 0.2635 & 0.0388 & 0.9981 \\
QESD product & 0.3268 & 0.0369 & 0.9985 \\
\hline
\end{tabular}




\subsubsection{Determination of the Residual Solvents}

As shown in Table 3, the amount of residual organic solvents is very low. Since all the organic solvents and surfactant used in this work are hydrosoluble or volatile, these solvents can be efficiently removed by washing and drying. The final products were azithromycin dihydrate, therefore the residual water is consistent with the theoretical amount of water in azithromycin dehydrate $(4.64 \%)$. For the residual acetone and ethyl acetate, the pharmaceutical industry requirements are less than $0.5 \%$, which are higher than residual acetone $(0.18 \%)$ and ethyl acetate $(0.17 \%)$ in spherical particles. In addition, these solvents and tween 80 are often used in the production of drugs and food. Hence, the spherical particles prepared by these methods can meet the requirements of drug safety.

Table 3. Purity and residual solvent of spherical crystallization products.

\begin{tabular}{cccccc}
\hline \multirow{2}{*}{ Product Type } & \multirow{2}{*}{$\begin{array}{c}\text { Azithromycin } \\
\text { Purity, wt } \%\end{array}$} & \multicolumn{4}{c}{ Residual Solvent, wt\% } \\
\cline { 3 - 6 } & Water & Acetone & Ethyl Acetate & Tween80 \\
\hline Commercial product & 95.05 & 4.73 & 0.12 & - & - \\
SA product & 94.95 & 4.81 & 0.18 & 0.13 & - \\
QESD product & 94.81 & 4.92 & - & 0.17 & 0.008 \\
\hline
\end{tabular}

\subsection{Evaluation of Tablet Properties}

In order to verify that spherical particles are suitable for direct tableting, tablet hardness, compaction curves, tablet content uniformity and disintegration time were investigated systematically. The results of hardness, friability, disintegration time, weight and content uniformity of azithromycin tablets are listed in Table 4. The compaction curve is shown in Figure S3 in the Supporting Information.

Table 4. Hardness, friability, disintegration time, tablet weight and content uniformity of azithromycin tablets.

\begin{tabular}{cccc}
\hline Tablet Properties & Commercial Product & SA Product & QESD Product \\
\hline Hardness (N) $^{\mathrm{a}}$ & $70.0 \pm 0.26$ & $85.0 \pm 0.38$ & $82.0 \pm 0.32$ \\
Friability (\%) $^{\mathrm{a}}$ & $1.35 \pm 0.56$ & $0.78 \pm 0.41$ & $0.83 \pm 0.45$ \\
Content uniformity (\%) $^{\mathrm{a}}$ & $96.6 \pm 4.1$ & $99.8 \pm 1.31$ & $99.2 \pm 1.56$ \\
Tablet weight (mg) $^{\mathrm{a}}$ & $489 \pm 2.31$ & $498 \pm 1.27$ & $496 \pm 1.69$ \\
Disintegration time (Min) $^{\mathrm{a}}$ & $8.23 \pm 1.15$ & $7.36 \pm 0.86$ & $6.79 \pm 1.72$ \\
\hline
\end{tabular}

${ }^{\mathrm{a}}$ All the values are shown as mean \pm standard deviation.

It can be seen from Table 4 that the tablet hardness and tensile strength of the tablets made of spherical particles were increased. This result should be attributed to the unique structure of spherical particles which consist of extremely small crystals. During the early stage of the compression process, spherical particles will be crushed, which will increase the interparticulate contact surface, thus leading to the increase of tensile strength [34,35]. The tablet friability of spherical particles can also meet the requirement $(<1 \%)$ due to the enhancement of tablet hardness. Since the flowability of spherical products were enhanced, the tablet content and weight uniformity were improved. Additionally, the disintegration time of spherical products decreased. This should be explained by the fact that the tablets made of spherical particles usually have a large porosity. To verity this, the porosity of the tablets was measured. It was demonstrated that the porosities of the tablets made of SA product $(23 \%)$ and QESD product $(27 \%)$ were larger than that of the tablets made of commercial product $(17 \%)$. Thereby, the disintegration time of spherical products decreased. Furthermore, as shown in Figure 15, owing to the large porosity, the dissolution rate of the tablets made of spherical particles was slightly faster than that of the tablets made of commercial product. To summarize, tablets made of spherical particles exhibited excellent tableting properties. Therefore, these spherical particles can be applied for direct tableting. 

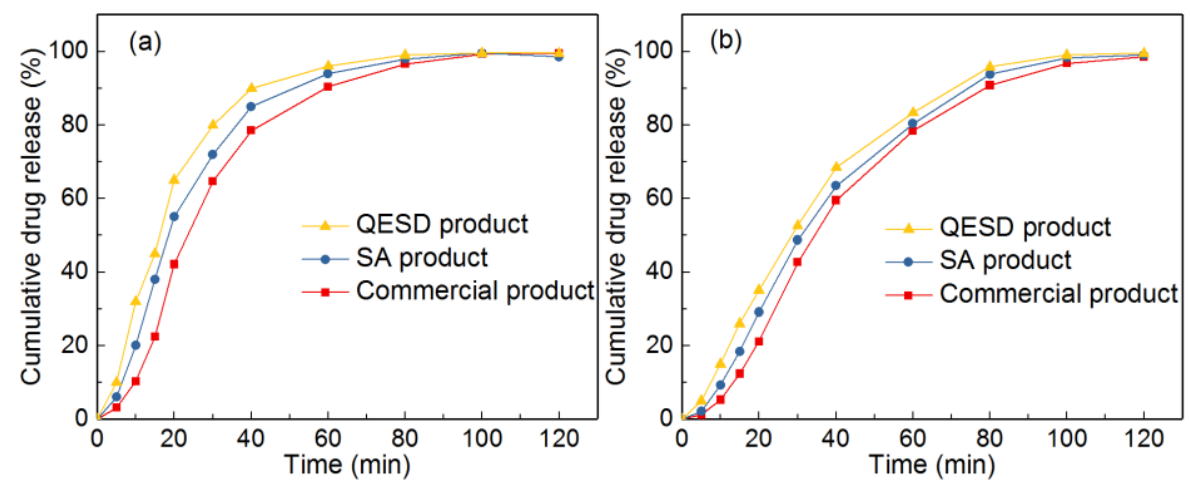

Figure 15. Dissolution profiles of the commercial product, SA product and QESD product at different pH: (a) $\mathrm{pH} 1.0 ;(\mathbf{b}) \mathrm{pH} 6.0$.

\section{Conclusions}

Two different spherical crystallization technologies were developed and utilized to successfully make solid and hollow spherical crystals of azithromycin. The size of spherical crystals was controllable and exhibited a narrow distribution. Compared with commercial product, the flowability, packability and particle size of spherical crystals were significantly improved. For the solid spheres, an immersion mechanism was proposed to explain the formation processes of spherical particles with the help of PAT tools. For hollow spheres, the counter diffusion of good solution and anti-solvent on the surface of droplets was proposed to be the formation mechanism. Many parameters will affect the formation process and the quality of the final products. By evaluating the tablet properties, it was proved that the properties of tablets made of spherical particles were improved.

Supplementary Materials: The following are available online at http://www.mdpi.com/2227-9717/7/5/276/s1, Figure S1: SEM images of the structure units in spherical particles, Table S1: Experimental conditions for SA process, Table S2: experimental conditions for QESD process, Figure S2: Packability curve of commercial and spherical products, Figure S3: Compaction curves of commercial and spherical products.

Author Contributions: Conceptualization, K.C. and H.H.; methodology, K.C.; formal analysis, B.H., X.H. and J.L.; investigation, K.C. and X.H.; writing-original draft preparation, K.C.; writing-review and editing, H.H., F.L., Y.X. and J.L.; visualization, H.H., Y.B. and H.W.; supervision, H.H. and X.H.; project administration, K.C. and H.H.; funding acquisition H.H.

Funding: This research was funded by National Natural Science Foundation of China, grant number 51478308.

Conflicts of Interest: The authors declare no conflict of interest.

\section{References}

1. Ferguson, S.; Ortner, F.; Quon, J.; Peeva, L.; Livingston, A.; Trout, B.L.; Myerson, A.S. Use of Continuous MSMPR Crystallization with Integrated Nanofiltration Membrane Recycle for Enhanced Yield and Purity in API Crystallization. Cryst. Growth Des. 2014, 14, 617-627. [CrossRef]

2. Jung, J.-W.; Kim, K.-J. Effect of Supersaturation on the Morphology of Coated Surface in Coating by Solution Crystallization. Ind. Eng. Chem. Res. 2011, 50, 3475-3482. [CrossRef]

3. Barrett, M.; McNamara, M.; Hao, H.; Barrett, P.; Glennon, B. Supersaturation tracking for the development, optimization and control of crystallization processes. Chem. Eng. Res. Des. 2010, 88, 1108-1119. [CrossRef]

4. Lu, J.; Rohani, S. Polymorphism and Crystallization of Active Pharmaceutical Ingredients (APIs). Curr. Med. Chem. 2009, 16, 884-905. [CrossRef] [PubMed]

5. Huang, Y.; Jiang, Y.; Yang, X.; Ren, Y.; Zhan, D.; Cölfen, H.; Hou, Z.; Liu, X.Y. Direct Growth of Microspheres on Amorphous Precursor Domains in Polymer-Controlled Crystallization of Indomethacin. Cryst. Growth Des. 2016, 16, 1428-1434. [CrossRef]

6. Yazdanpanah, N.; Langrish, T.A.G. Crystallization and Drying of Milk Powder in a Multiple-Stage Fluidized Bed Dryer. Drying Technol. 2011, 29, 1046-1057. [CrossRef] 
7. Barrett, M.; Hao, H.; Maher, A.; Hodnett, K.; Glennon, B.; Croker, D. In Situ Monitoring of Supersaturation and Polymorphic Form of Piracetam during Batch Cooling Crystallization. Org. Process Res. Dev. 2011, 15, 681-687. [CrossRef]

8. Gyulai, O.; Szabó-Révész, P.; Aigner, Z. Comparison Study of Different Spherical Crystallization Methods of Ambroxol Hydrochloride. Cryst. Growth Des. 2017, 17, 5233-5241. [CrossRef]

9. Chattoraj, S.; Sun, C.C. Crystal and Particle Engineering Strategies for Improving Powder Compression and Flow Properties to Enable Continuous Tablet Manufacturing by Direct Compression. J. Pharm. Sci. 2018, 107, 968-974. [CrossRef]

10. Jivraj, M.; Martini, L.G.; Thomson, C.M. An overview of the different excipients useful for the direct compression of tablets. Pharm. Sci. Technol. Today 2000, 3, 58-63. [CrossRef]

11. Chen, H.; Wang, C.; Sun, C.C. Profoundly Improved Plasticity and Tabletability of Griseofulvin by in Situ Solvation and Desolvation during Spherical Crystallization. Cryst. Growth Des. 2019, 19, 2350-2357. [CrossRef]

12. Kawashima, Y.; Okumura, M.; Takenaka, H. Spherical Crystallization: Direct Spherical Agglomeration of Salicylic Acid Crystals During Crystallization. Science 1982, 216, 1127. [CrossRef]

13. Chatterjee, A.; Gupta, M.M.; Srivastava, B. Spherical crystallization: A technique use to reform solubility and flow property of active pharmaceutical ingredients. Int. J. Pharm. Investig. 2017, 7, 4-9.

14. Zhang, Z.-B.; Shen, Z.-G.; Wang, J.-X.; Zhang, H.-X.; Zhao, H.; Chen, J.-F.; Yun, J. Micronization of silybin by the emulsion solvent diffusion method. Int. J. Pharm. 2009, 376, 116-122. [CrossRef]

15. Zhang, D.; Tan, T.; Gao, L.; Zhao, W.; Wang, P. Preparation of Azithromycin Nanosuspensions by High Pressure Homogenization and its Physicochemical Characteristics Studies. Drug Dev. Ind. Pharm. 2007, 33, 569-575. [CrossRef] [PubMed]

16. Mohammadi, G.; Valizadeh, H.; Barzegar-Jalali, M.; Lotfipour, F.; Adibkia, K.; Milani, M.; Azhdarzadeh, M.; Kiafar, F; Nokhodchi, A. Development of azithromycin-PLGA nanoparticles: Physicochemical characterization and antibacterial effect against Salmonella typhi. Colloids Surf. B 2010, 80, 34-39. [CrossRef]

17. Adeli, E. The use of spray freeze drying for dissolution and oral bioavailability improvement of Azithromycin. Powder Technol. 2017, 319, 323-331. [CrossRef]

18. Peña, R.; Jarmer, D.J.; Burcham, C.L.; Nagy, Z.K. Further Understanding of Agglomeration Mechanisms in Spherical Crystallization Systems: Benzoic Acid Case Study. Cryst. Growth Des. 2019, 19, 1668-1679. [CrossRef]

19. Mukhopadhyay, I.; Mohandas, V.P.; Desale, G.R.; Chaudhary, A.; Ghosh, P.K. Crystallization of Spherical Common Salt in the Submillimeter Size Range without Habit Modifier. Ind. Eng. Chem. Res. 2010, 49, 12197-12203. [CrossRef]

20. Jitkar, S.; Thipparaboina, R.; Chavan, R.B.; Shastri, N.R. Spherical Agglomeration of Platy Crystals: Curious Case of Etodolac. Cryst. Growth Des. 2016, 16, 4034-4042. [CrossRef]

21. Wu, H.; Wang, J.; Xiao, Y.; Huang, X.; Guo, Y.; Zong, S.; Liu, Q.; Hao, Y.; Hao, H. Manipulation of Crystal Morphology of Zoxamide Based on Phase Diagram and Crystal Structure Analysis. Cryst. Growth Des. 2018, 18, 5790-5799. [CrossRef]

22. Lamešić, D.; Planinšek, O.; Lavrič, Z.; Ilić, I. Spherical agglomerates of lactose with enhanced mechanical properties. Int. J. Pharm. 2017, 516, 247-257. [CrossRef] [PubMed]

23. Maghsoodi, M.; Esfahani, M. Preparation of microparticles of naproxen with Eudragit RS and Talc by spherical crystallization technique. Pharm. Dev. Technol. 2009, 14, 442-450. [CrossRef] [PubMed]

24. Nokhodchi, A.; Maghsoodi, M.; Hassanzadeh, D. An Improvement of Physicomechanical Properties of Carbamazepine Crystals. Iran. J. Pharm. Res. 2010, 6, 83-93.

25. Curatolo, W.; Liu, P.; Johnson, B.A.; Hausberger, A.; Quan, E.; Vendola, T.; Vatsaraj, N.; Foulds, G.; Vincent, J.; Chandra, R. Effects of Food on a Gastrically Degraded Drug: Azithromycin Fast-Dissolving Gelatin Capsules and HPMC Capsules. Pharm. Res. 2011, 28, 1531-1539. [CrossRef]

26. Adeli, E. A comparative evaluation between utilizing SAS supercritical fluid technique and solvent evaporation method in preparation of Azithromycin solid dispersions for dissolution rate enhancement. J. Supercrit. Fluids 2014, 87, 9-21. [CrossRef]

27. Wu, S.; Shen, H.; Li, K.; Yu, B.; Xu, S.; Chen, M.; Gong, J.; Hou, B.H. Agglomeration Mechanism of Azithromycin Dihydrate in Acetone-Water Mixtures and Optimization of the Powder Properties. Ind. Eng. Chem. Res. 2016, 55, 4905-4910. [CrossRef] 
28. Schæfer, T.; Mathiesen, C. Melt pelletization in a high shear mixer. IX. Effects of binder particle size. Int. J. Pharm. 1996, 139, 139-148. [CrossRef]

29. Pitt, K.; Peña, R.; Tew, J.D.; Pal, K.; Smith, R.; Nagy, Z.K.; Litster, J.D. Particle design via spherical agglomeration: A critical review of controlling parameters, rate processes and modelling. Powder Technol. 2018, 326, 327-343. [CrossRef]

30. Jiang, Y.; Gower, L.; Volkmer, D.; Cölfen, H. Hierarchical DL-Glutamic Acid Microspheres from Polymer-Induced Liquid Precursors. Cryst. Growth Des. 2011, 11, 3243-3249. [CrossRef]

31. Peña, R.; Nagy, Z.K. Process Intensification through Continuous Spherical Crystallization Using a Two-Stage Mixed Suspension Mixed Product Removal (MSMPR) System. Cryst. Growth Des. 2015, 15, 4225-4236. [CrossRef]

32. Cui, F.; Yang, M.; Jiang, Y.; Cun, D.; Lin, W.; Fan, Y.; Kawashima, Y. Design of sustained-release nitrendipine microspheres having solid dispersion structure by quasi-emulsion solvent diffusion method. J. Control. Release 2003, 91, 375-384. [CrossRef]

33. Kawashima, Y.; Capes, C.E. Further studies of the kinetics of spherical agglomeration in a stirred vessel. Powder Technol. 1976, 13, 279-288. [CrossRef]

34. Thakur, A.; Thipparaboina, R.; Kumar, D.; Sai Gouthami, K.; Shastri, N.R. Crystal engineered albendazole with improved dissolution and material attributes. Cryst. Eng. Comm. 2016, 18, 1489-1494. [CrossRef]

35. Nokhodchi, A.; Maghsoodi, M.; Hassan-Zadeh, D.; Barzegar-Jalali, M. Preparation of agglomerated crystals for improving flowability and compactibility of poorly flowable and compactible drugs and excipients. Powder Technol. 2007, 175, 73-81. [CrossRef]

(C) 2019 by the authors. Licensee MDPI, Basel, Switzerland. This article is an open access article distributed under the terms and conditions of the Creative Commons Attribution (CC BY) license (http://creativecommons.org/licenses/by/4.0/). 